\title{
A Brief Review on Dynamic Reliable Location Aware Routing Protocols for Mobile Wireless Sensor Network
}

\author{
Simerjeet \\ M.Tech. Student, Department of Computer \\ Science and Engineering \\ SGGSW University, Fatehgarh Shaib.
}

\author{
Navpreet Kaur \\ Assistant Prof., Department of Computer Science \\ and Engineering \\ SGGSW University, Fatehgarh Shaib
}

\begin{abstract}
Energy efficiency, network lifetime, data transmission, endto-end delay and reliable routing protocols for mobility centric are the major parameters used for wireless sensor network (WSN), and are maintained by various of protocols. Existing cluster-based mobile routing protocols such as LEACH, LEACH-Mobile, LEACH-Mobile Enhanced, CBR-Mobile, LFCP-MWSN consider energy efficiency of sensor node. These protocols allocate the extra timeslots using time division multiple access (TDMA) scheme to accommodate nodes that enter a cluster because of mobility and thus increase the end-to-end delay. In this paper we discussed the various protocols such as LEACH, LEACH-M, LEACH-ME, DSC, CBR-M, LFCP-MWSN. The results revealed that that LFCP-MWSN protocol is more efficient in terms of energy consumptions, network lifetime and data transmissions than those of the existing LEACH-M and LEACH-ME protocols.
\end{abstract}

\section{Keywords}

LEACH; Clustering Methods; Wireless Sensor

Network(WSN).

\section{INTRODUCTION}

Wireless sensor networks (WSNs) consist of hundreds and thousands of unattended, resource-constraint and low-energy sensor nodes designing energy efficient routing protocols is significantly important. Clustering-based routing protocols are more useful in the context of energy efficiency where several sensor nodes in the communication range of one another form a cluster. Each cluster has a cluster head $(\mathrm{CH})$, which coordinates all the nodes of a cluster. There may be a number of base stations (BS) also known as sink in a WSN that communicate with other networks [1, 2]. Most clustering protocols of WSN in the literature are designed for static sensor nodes. Thus, these protocols do not work for WSN applications that require mobile sensor nodes, such as habitat monitoring, wild life monitoring, target tracking and battlefield surveillance. Moreover, these protocols do not support localization of sensor nodes but only assume that each node know their location, which make these protocols inefficient. For instance, low energy adaptive clustering hierarchy (LEACH) Protocol [3] is a standard static clustering protocol of WSN. LEACH is enhanced as LEACH-Mobile [4], LEACH-Mobile-Enhancement [5], and cluster based routing protocol for mobile nodes in wireless sensor network (CBR Mobile-WSN) [1] to support mobility of sensor nodes. These protocols work in rounds and initiate a new cluster formation phase at every round, where each round comprises cluster formation, $\mathrm{CH}$ selection and data transmission phases. This is also not considered energy efficient since a large number of messages are transmitted to form a cluster. To alleviate this problem propose a new location aware fault tolerant clustering protocol for mobile WSN (LFCP-MWSN). In this protocol, a special packet is sent by a non- $\mathrm{CH}$ node $\mathrm{A}$ if $\mathrm{A}$ has no sensed data to send to the $\mathrm{CH}$ at its allocated timeslot and thus, saves energy by not sending data at every timeslot. At the end of around a node with the least mobility is selected as a new $\mathrm{CH}$, which is calculated as the ratio of the number movements of a node inside and outside of its cluster. In addition to this, LFCP-MWSN supports sensors localization in the cluster formation phase and every time a node moves to a new location since without location information sensors data are meaningless for most of the applications. It also consumes energy but slightly less than other protocols. In this $\mathrm{CH}$ does not moved it is stationary due to this it consume more energy, high average delay and large number of packet loss $[8,14]$.

\section{VARIOUS APPROACHES}

- Low energy adaptive clustering Protocol (LEACH)

Low energy adaptive clustering Protocol (LEACH) [2] works well for homogeneous networks, where every node has the same initial energy. This protocol works in rounds and each round is divided into cluster formation and steady phases. In the cluster formation phase, a cluster is formed and p.n sensor nodes are selected as cluster heads $(\mathrm{CH})$ for the proper utilization of energy, where $\mathrm{n}$ is the number of sensor nodes and $\mathrm{p}$ is the desired percentage of $\mathrm{CH}$. The steady state is divided into many frames where $\mathrm{CH}$ assigns time slots to each non- $\mathrm{CH}$ node using TDMA scheme. At the end of each round, the $\mathrm{CH}$ collects and aggregates data and sends to the BS.

Once the nodes have elected themselves to be cluster heads using the probabilities in (3) or (6), the cluster head nodes must let all the other nodes in the network know that they have chosen this role for the current round. To do this, each cluster head node broadcasts an advertisement message (ADV) using a nonpersistent carrier-sense multiple access (CSMA) MAC protocol [12]. This message is a small message containing the node's ID and a header that distinguishes this message as an announcement message. Each non-cluster head node determines its cluster for this round by choosing the cluster head that requires the minimum communication energy, based on the received signal strength of the advertisement from each cluster head. Assuming symmetric propagation channels for pure signal strength, the cluster head advertisement heard with the largest signal strength is the cluster head that requires the minimum amount of transmit energy to communicate with. 


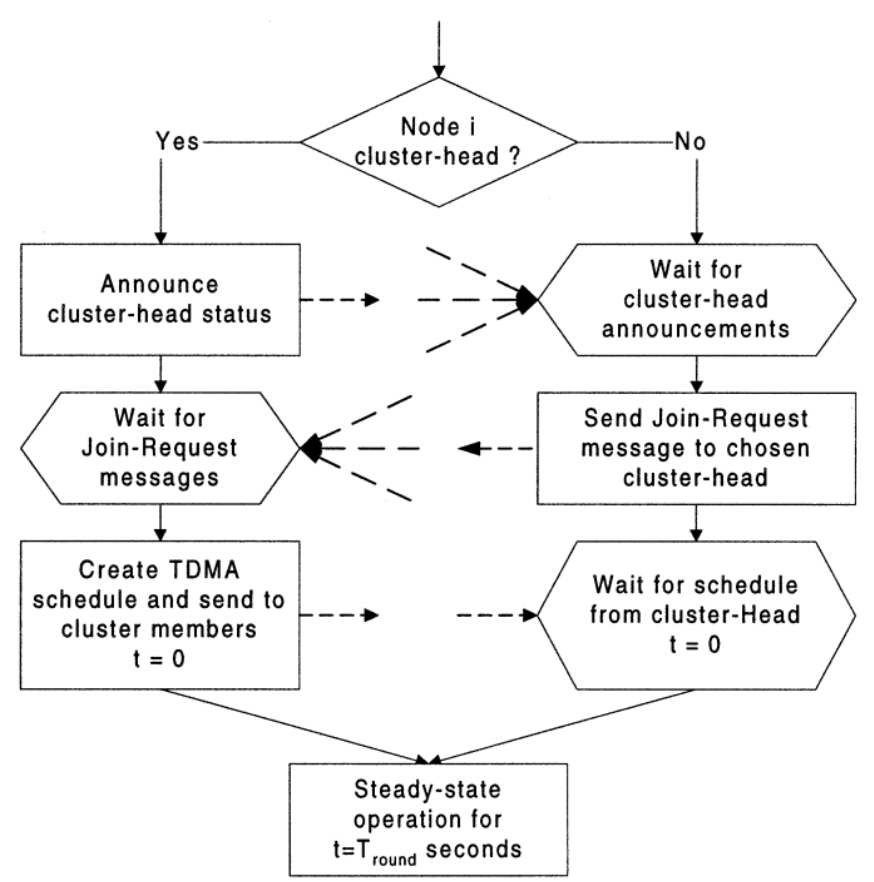

Fig.1. Flowchart of the distributed cluster formation algorithm for LEACH [12]

In the LEACH protocol, a new cluster formation is initiated in every round. All $\mathrm{CHs}$ exist in a close area so it $\mathrm{h}$ as less chances to loss the data packet. LEACH Require more energy for non- $\mathrm{CH}$ nodes to communicate $\mathrm{CHs}$. A new cluster formation is not energy efficient in the LEACH protocol. LEACH Protocol is energy efficient, network lifetime and good for communication overhead.

\section{- Dynamic Static Clustering Protocol (DSC)}

In [6], Bajaber and Awan proposed dynamic static clustering protocol (DSC) for (WSN). Dynamic case is divided into two phases: Setup and Steady phase. In the Setup phase, the base station (BS) forms clusters and selects $\mathrm{CH}$ for each cluster based on the energy levels and positions of the sensor nodes. Then, the BS broadcasts CH ID to all nodes. A sensor node will be a CH if its ID matches with the CH ID. In the Steady phase, $\mathrm{CH}$ uses TDMA scheme by dividing each frame into $\mathrm{x}$ number of timeslots, where $\mathrm{x}$ is the total number of non- $\mathrm{CH}$ nodes in that cluster. Static case has only the steady phase, which is similar to that of dynamic case except for after a certain number of rounds (i.e. 10) a new cluster formation/setup phase is initiated. However, the static case has less number of cluster formation phases as compared to the dynamic case and so, has less transmission overhead.DSC has better performance than LEACH in terms of energy efficiency, large network lifetime. DSC is better than LEACH in the communication overhead. DSC does not provide mobility of sensor nodes. It cannot be used in applications habitat monitoring, target tracking.

\section{- Low energy adaptive clustering Protocol- Mobile}

To provide mobility of sensor nodes, Kim and Chung [7] proposed LEACH-Mobile (LEACH-M) routing protocol. LEACH-Mobile (LEACH-M) routing protocol where cluster formation and $\mathrm{CH}$ selection mechanism is same as LEACH. LEACH-M ensures the communication of a node with a $\mathrm{CH}$ even if node is in motion by transmitting data request packet from $\mathrm{CH}$ to the sensor node in its allocated timeslot using TDMA scheme. LEACH-M handles node mobility by assuming that the $\mathrm{CHs}$ are stationary. LEACH-M ensures the communication of a node with a CH. It has also a JOIN-ACK to join in a new cluster. LEACH-M is not considered in term of energy consumptions and data delivery rate because a large number of packet are lost if the $\mathrm{CH}$ keeps moving before selecting a new $\mathrm{CH}$ for the next round. LEACH-M handles node mobility by assuming that the $\mathrm{CHs}$ are stationary. It ensures the communication of a node with $\mathrm{CH}$. Successful data transmissions in different mobility factors. And reduce the chance of data loss in case of node's mobility. To alleviate this problem of LEACH-M, Kumar et al. propose LEACHMobile-Enhanced (LEACH-ME), LEACH-Mobile-Enhanced (LEACH-ME) where a node with the minimal mobility factor is selected as a $\mathrm{CH}$, if the residual energy of the node is not below a threshold value. They calculate mobility factor based on the number of times a node moves from a cluster to another cluster. Since mobility factor is a function of distance among nodes it is calculated by multiplying node's velocity with the time required to move a node from a position to another. For example, node i can make use of IDs of all other nodes it hears and calculate $\operatorname{dij}(\mathrm{t})$ as $\operatorname{dij}(\mathrm{t})=$ RadioVelocity $* \mid \mathrm{t} 2$ $\mathrm{t} 1$, where at time $\mathrm{t} 1$ node $\mathrm{i}$ broadcast its ID and at time $\mathrm{t} 2$ it receives the ID of node $\mathrm{j}$. LEACH-ME provides a minimal data loss in case of node's mobility. It is better than LEACH$M$ in successful data transmissions in different mobility factors. In the LEACH-ME Wastages of timeslots and it is the energy consumption. The performance of LEACH-ME is better than LEACH-M in successful data transmissions in different mobility factors. LEACH-M is not energy efficient since it consumes energy for determining mobility factors in active slots.

\section{- Cluster Based Routing Protocol}

Awwad et al. proposed [1] cluster based routing protocol for mobile nodes in WSN (CBR Mobile-WSN). CBR-M is an adaptive protocol that avoids wastage of timeslots and hence, ensures efficient bandwidth utilization. Each $\mathrm{CH}$ keeps some free timeslots to enable other incoming mobile nodes from other clusters to join its cluster. A $\mathrm{CH}$ sends data request message to the non- $\mathrm{CH}$ nodes and if the $\mathrm{CH}$ does not receive data from a member, the packet is considered to be lost and the $\mathrm{CH}$ discards the nodes membership, at the end of the frame. CBR-M routing protocol avoid the wastage of timeslots. It reduces the energy consumption and also reduces the chance of the packet loss. CBR-M has more average delay. Cluster Based Routing Protocol for Mobile Node in WSN to reduce energy consumption and the number of packets loss.

\section{- Location-aware and Fault tolerant Clustering Protocol}

L. Karim, N. Nasser [8], proposed LFCP-MWSN routing protocol in this all sensors are mobile .Once a node is selected as a $\mathrm{CH}$, it remains in the same cluster. Initially, all sensors have the same energy. A node in each cluster is equipped with GPS and work only for localization. This node is known as anchors node. Sensors are heterogeneous in terms of their roles since they work as anchor nodes, cluster heads, and cluster members. LFCP-MWSN Protocol using C programming language.

Initially, (BS) divides the network into a number of clusters based on the geographical locations of sensors, assigns ID to clusters and sensors. Then sensors are localized using the technique that is presented and. Then BS selects $\mathrm{CHs}$ based on the initial node energy and position of the sensors. Since initially all nodes have the same energy, $\mathrm{CH}$ is randomly selected based on a random number between 0 and 1 and $\mathrm{CH}$ 
probability, which is similar to the method used in the LEACH protocol $[2,6]$. Then $\mathrm{CHs}$ broadcast their positions and IDs. A node $\mathrm{A}$ is assigned to a cluster whose $\mathrm{CH}$ is at the minimum distance with $\mathrm{A}$. The node $\mathrm{A}$ then sends a registration message to the $\mathrm{CH}$ with its ID and current location. All clusters' information is then sent to BS for centralized control and operations. Once a $\mathrm{CH}$ is selected at the beginning of a round it is considered to be static until a new $\mathrm{CH}$ is selected in the next round based on the mobility factor of sensor nodes. After a number of rounds a new cluster formation and $\mathrm{CH}$ selection phase (based on nodes mobility) is initiated to balance the energy consumptions. Once the network operation starts and nodes move at a fixed and low velocity, each node keeps track of the number of movements inside and outside of its current cluster based on which nodes mobility is calculated at each round.

In the steady phase, given fig 1.CHs assign timeslots to the member nodes using TDMA scheme. Member nodes of a cluster transmit data, receive acknowledgement from $\mathrm{CH}$ and count their movement inside and outside of the cluster at the allocated timeslot. Thus, no extra timeslot is required to calculate nodes mobility. However, one extra timeslot is assigned in each frame to allow a mobile node to send JOIN REQUEST message to the $\mathrm{CH}$ of a new cluster when that node moves out of a cluster. Existing mobile routing protocols mention that mobile nodes join in a new $\mathrm{CH}$ by sending a join request packet but do not mention how this join request message is transmitted in the TDMA scheme since timeslots are all allocated only to the cluster member nodes. We also assume that all nodes are homogeneous in terms of mobility and so, while a node moves out of a cluster there is a high probability of another node entering into that cluster. However, if a node moves into a new cluster and sends JOINREQUEST message to $\mathrm{CH}$ using the free timeslot, the $\mathrm{CH}$ does not allocate the node a timeslot until any timeslot becomes free for moving a node out of this cluster. If a $\mathrm{CH}$ does not receive any data or special packet from node $\mathrm{A}$ at its allocated timeslot the $\mathrm{CH}$ assumes that (i) data or special packet transmission has failed or (ii) the node A has moved out of its cluster or (iii) node A has failed. To confirm about the transmission failure $\mathrm{CH}$ waits until the next timeslot of node $\mathrm{A}$. If $\mathrm{CH}$ does not receive any data or special packet in the next timeslot $\mathrm{CH}$ deletes node $\mathrm{A}$ from its members list and also the timeslot allocated to that node. $\mathrm{CH}$ also notifies BS the ID of A that it has either moved or died [8].

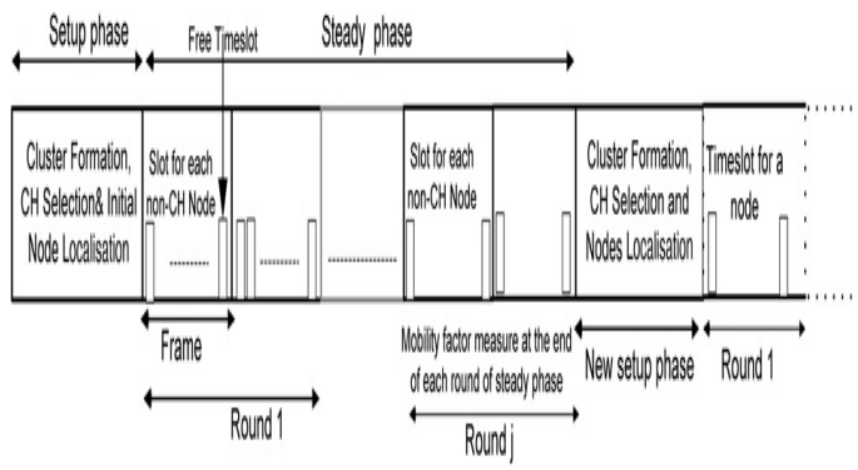

Fig. 2 Cluster formation and steady state [8]

A simple, light-weight and range-free localization approach is used in LFCP-MWSN Protocols. LFCP-MWSN protocol provide fault tolerance by using small sized special packets. The transmission energy consumptions of node in LFCPMWSN protocol are less. The performance of the LFCPMWSN protocol in terms of energy consumptions, lifetime, end-to-end delay and number of communications. LFCPMWSN protocol has end-to-end delay but as compare other protocols it has less. A LFCP-MWSN that supports mobility of sensor nodes and sensors localization. Simulation results show that LFCP-MWSN protocol is more efficient in terms of energy consumptions, network lifetime and data transmissions.

\section{CONCLUSION}

LFCP-MWSN protocol is more efficient in term of energy consumption, network lifetime and data transmission than those of existing LEACH-M and LEACH-ME protocols. LEACH and DSC protocols have no mobility of sensor nodes. Moreover, LFCP-MWSN detects the failure of sensor nodes. Although the analysis shows that LFCP-MWSN protocol should have less end-to-end network delay and packet loss than LEACH-M and LEACH-ME. LFCP-MWSN protocol, we consider that once a node with the least mobility factor is selected as a $\mathrm{CH}$, then the $\mathrm{CH}$ will not move out of the cluster in the current round. Clustering in mobile WSN provide vast range of dynamic behavior as well as improvement in the performance in the system parameters

\begin{tabular}{|c|c|c|c|c|c|c|}
\hline $\begin{array}{l}\text { Protocols/ } \\
\text { Parameters }\end{array}$ & LEACH & DSC & LEACH-M & LEACH-ME & CBR-M & LFCP-MWSN \\
\hline ENERGY CONSUMPTION & $\begin{array}{l}\text { VERY } \\
\text { HIGH }\end{array}$ & MEDIUM & HIGH & MEDIUM & MEDIUM & MEDIUM \\
\hline NETWORK LIFETIME & LOW & MEDIUM & LOW & VERY LOW & MEDIUM & MEDIUM \\
\hline $\begin{array}{l}\text { COMMUNICATION } \\
\text { OVERHEAD }\end{array}$ & LOW & MEDIUM & LOW & LOW & LOW & MEDIUM \\
\hline TIME SLOT & LOW & LOW & LOW & VERY LOW & LOW & LOW \\
\hline MOBILITY SESOR NODE & $\mathrm{NO}$ & $\mathrm{NO}$ & YES & YES & YES & YES \\
\hline NO. OF PACKET LOSS & HIGH & HIGH & HIGH & HIGH & MEDIUM & MEDIUM \\
\hline AVERAGE DELAY & HIGH & HIGH & HIGH & HIGH & HIGH & HIGH \\
\hline
\end{tabular}




\begin{tabular}{|l|l|l|l|l|l|l|}
\hline CLUSTER HEAD MOVE & NO & NO & NO & NO & NO & NO \\
\hline
\end{tabular}

\section{Table for Comparison of Protocols}

\section{REFERENCES}

[1] Awwad, S.A.B., Ng, C.K., Noordin, N.K., Rasid, M.F.A., "Cluster based routing protocol for mobile nodes in wireless sensor network", International Conference on Collaborative Technologies and Systems (CTS), pp. 233-241, 2009.

[2] Durresia, A., Paruchuri, V., Barolli, L., "Clustering protocol for sensor networks",20th International Conference on Advanced Information Networking and Applications (AINA), Vol. 2, 2006

[3] Kumar, G.S., Vinu , M.V., Athithan, P.G., Jacob, K.P., "Routing protocol enhancement for handling node mobility in wireless sensor networks", IEEE Region Conference, 2009.

[4] Zheng, G.-p., Zhou, Y., "An energy-aware cluster protocol for wireless sensor networks", Second International Conference on Innovative Computing, Information and Control (ICICIC), Vol. 7, N0. 10, pp. 473-473, 2012.

[5] Huang, B., Hao, F., Zhu, H., Tanabe, Y., Baba, T., "Low-energy static clustering scheme for wireless sensor network", International Conference on Wireless Communications, Networking and Mobile Computing (WiCOM), 2006.

[6] Bajaber, F., Awan, I., "Dynamic/static clustering protocol for wireless sensor network", Second UKSIM European Symp. on Computer Modeling and Simulation IEEE, Vol. 8, pp. 524-529, 2008.

[7] Kim, D.-S., Chung, Y.-J., "Self-organization routing protocol supporting mobile nodes for wireless sensor network", First International Multi-Symp. On Computer and Computational Sciences, pp. 221-234, 2006.

[8] L.Karim, N.Nasser, "Reliable location-aware routing protocol for mobile wireless sensor network",
International Conference on the Institution of Engineering and Technology, Vol. 6, No. 14, pp. 21492150, 2012.

[9] Seema Bandyopadhyay and Edward J. Coyle, "An Energy Efficient Hierarchical Clustering Algorithm for Wireless Sensor Networks", IEEE Region Conference, pp. 1713-1723, 2003.

[10] I.F. Akyildiz, W. Su, Y. Sankarasubramaniam, E. Cayirci , "Wireless sensor networks: a survey", Elsevier Science B.V., Vol. 38, No. 4, pp. 393-422, 2002.

[11] Li Qing, Qingxin Zhu, Mingwen Wang, "Design of a distributed energy-efficient clustering algorithm for heterogeneous wireless sensor networks", Elsevier B.V. All rights reserved, pp. 2230-2237, 2006.

[12] Wendi B. Heinzelman, Anantha P. Chandrakasan, and Hari Balakrishnan, "An Application-Specific Protocol Architecture for Wireless Microsensor Networks", IEEE transaction on wireless communications, Vol. 1, NO. 4, pp. 660-670, 2002.

[13] JAMAL N. AL-KARAKI, AHMED E. KAMAL, "Routing Techniques in Wireless Sensor Networks: A Survey", International Conference of IEEE Wireless Communications, pp. 6-28, 2004.

[14] Shashidhar Rao Gandham, Milind Dawande, Ravi Prakash and S. Venkatesan, "Energy Efficient Schemes for Wireless Sensor Networks with Multiple Mobile Base Stations”, IEEE Conference, pp. 377-381, 2003.

[15] Kisuk Kweon, Hojin Ghim, Jaeyoung Hong and Hyunsoo Yoon, "Grid-Based Energy-Efficient Routing from Multiple Sources to Multiple Mobile Sinks in Wireless Sensor Networks", IEEE Conference, pp. 1-5, 2009. 